\title{
EFFECTS OF MAGNETIC FIELD AND NON-UNIFORM BASIC TEMPERATURE GRADIENT ON THE ONSET OF RAYLEIGH-BENARD CONVECTION IN A MICROPOLAR FLUID
}

\author{
S. Pranesh*
}

\begin{abstract}
The effects of magnetic field and non-uniform basic temperature gradient on the onset of Rayleigh-Benard convection in an electrically conducting micropolar fluid are studied using the Galerkin technique. The eigenvalue is obtained for free-free, rigid-free and rigid-rigid velocity boundary combinations with isothermal and adiabatic temperature conditions on the spin-vanishing boundaries. The eigenvalues are also obtained for lower rigid isothermal and upper free adiabatic boundaries with vonishing spin.A linear stability analysis is performed. The influence of various parameters on the onset of convection has been analyzed.Six different non-uniform temperature profiles are considered and their comparative influence on onset is discussed. It is observed that the electrically conducting fluid layer with suspended particles heated from below is more stable compared to the classical electrically conducting fluid without suspended particles. The critical wave number is found to be insensitive to the changes in the parameters but sensitive to the changes in the Chandrasekhor number.
\end{abstract}

* Department of Mathematics, Christ College, Hosur Road, Bangalore - 560 029, INDIA. email: spranesh@hotmail.com, phone: +91-080-3324819,Fox:+91-080-5535863 


\section{Introduction}

The theory of Rayleigh-Benard instability of a horizontal liquid layer with free upper surface over a heated plate has many important applications in a number of engineering problems, such as in oil extraction from porous medium, energy storage in molten salts, and chemical engineering of paints, colloids and detergents. The instability of Rayleigh-Benard convection is due to the effect of the thermal buoyancy. Theoretical studies of the onset of convection in classical viscous fluids with nonuniform heating have been made by Currie [1] with isothermal boundaries and by Nield [2] with adiabatic boundaries and showed that in the case of piecewise linear temperature profile the onset of convection could occur at a smaller Rayleigh number than that of uniform heating. The non-uniform temperature gradient finds its origin in transient heating or cooling at the boundaries and as a result the basic temperature profile depends explicitly on position and time. This has to be determined by solving the coupled momentum and energy equations. This coupling also makes the problem very complicated.In the present study, therefore, we adopt a series of temperature profiles based on a simplification in the form of a quasi-static approximation (Currie [1], Lebon and Cloot [3] and Rudraiah et al [4]) that consists of freezing the temperature distribution at a given instant of time. In this method, we assume that the perturbation grows much faster than the initial state and hence freeze the initial state into some spatial distribution. This hypothesis is sufficient for our purpose because we are interested only in finding the conditions for the onset of convection. Even with these simplifications, the solutions to the variable-coefficients stability equations pose a problem because the temperature gradient varies with depth.

Micropolar fluid theories [5-7] describe some physical systems, which do not satisfy the Navier-Stokes equation. To explain the kinematics of such media two new variables should be added to the velocity. These variables are the spin, responsible for microrotations, and the microinertia tensor, which accounts for the atoms and molecules inside the macroscopic fluid particles. These fluids are able to describe the behaviour of suspensions, liquid crystals, blood, etc (see Power [8], Lukaszewicz [9] and Eringen [10]). Chandra [11] observed in his experiments that adding smoke particles to a loyer of gas could decrease the Rayleigh number at which convective motion commences. Since the particle spin associated with Eringen's theory could possibly be appropriate to the added dust situation described by Chandra [11], there may be dust, dirt, ice or raindrops, or other additives. Thus we believe that the Eringen micropolar convection model may be applicable to geophysical or industrial convection contexts. The Rayleigh-Benardsituation in Eringen's micropolar fluids has been investigated by many authors [12-25]. The main result from all these studies is that for heating from below stationary convection 
is the preferred mode. The effect of non-uniform basic temperature gradients on the onset of Rayleigh-Benard convection in a micropolarfluid has not been given any attention. Hence, the aim of this paper is to study the effect of magnetic field and non-uniform basic temperature gradients on Rayliegh-Benard convection with the object of understanding the control of convection. A single term Galerkin method expansion procedure is used here with the intention of obtaining reasonable results with minimum of mathematics.

\section{Mathematical Formulation:}

Consider an infinite horizontal layer of a Boussinesquian, electrically conducting fluid, with non-magnetic suspended particle, of depth ' $d$ ' permeated by an externally applied uniform magnetic field normal to the layer (see figure (1)). A cartesian coordinate system is taken with origin in the lower boundary and z-axis vertically

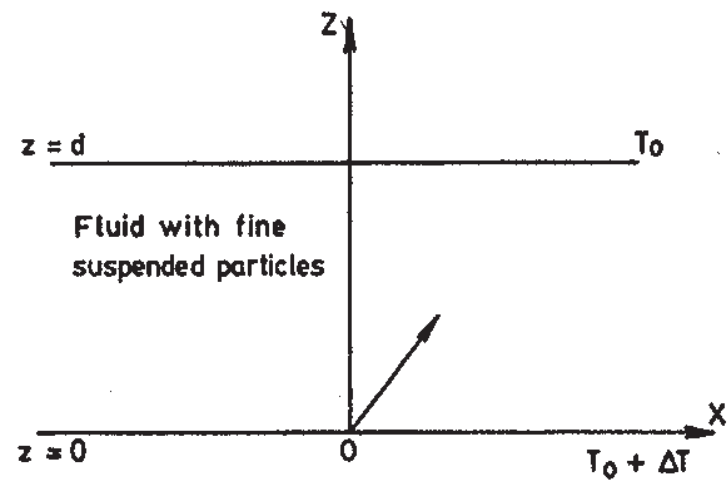

Fig. 1. Schematic Diagram of the Rayleigh-Benard situation for a fluid with suspended particles

upwards. Let DT be the temperature difference between the upper and lower boundaries. The body forces acting on the fluid are buoyancy and magnetic field. The governing equations for the Rayleigh-Benard situation in a Boussinesquian fluid with suspended particles are:

\section{Continuity equation:}

$\nabla \cdot \overrightarrow{\mathrm{q}}=0$ 


\section{Conservation of linear momentum:}

$\rho_{\mathrm{o}}\left[\frac{\partial \overrightarrow{\mathrm{q}}}{\partial \mathrm{t}}+(\overrightarrow{\mathrm{q}} . \nabla) \overrightarrow{\mathrm{q}}\right]=-\nabla \mathrm{P}-\rho \mathrm{g} \hat{\mathrm{k}}+(2 \zeta+\eta) \nabla^{2} \overrightarrow{\mathrm{q}}+\zeta \nabla \times \vec{\omega}+\mu_{\mathrm{m}}(\overrightarrow{\mathrm{H}} . \nabla) \overrightarrow{\mathrm{H}}$,

Conservation of angular momentum

$\rho_{0} \mathrm{I}\left[\frac{\partial \vec{\omega}}{\partial \mathrm{t}}+(\overrightarrow{\mathrm{q}} \cdot \nabla) \vec{\omega}\right]=\left(\lambda^{\prime}+\eta^{\prime}\right) \nabla(\nabla \cdot \vec{\omega})+\eta^{\prime} \nabla^{2} \vec{\omega}+\zeta(\nabla \times \overrightarrow{\mathrm{q}}-2 \vec{\omega})$,

Conservation of energy

$\frac{\partial \mathrm{T}}{\partial \mathrm{t}}+\left(\overrightarrow{\mathrm{q}}-\frac{\beta}{\rho_{0} \mathrm{C}_{\mathrm{V}}} \nabla \times \vec{\omega}\right) \nabla \mathrm{T}=\chi \nabla^{2} \mathrm{~T}$,

Equation of state

$\rho=\rho_{0}\left[1-\alpha\left(T-T_{0}\right)\right]$,

Magnetic induction equation

$\frac{\partial \overrightarrow{\mathrm{H}}}{\partial \mathrm{t}}+(\overrightarrow{\mathrm{q}} \cdot \nabla) \overrightarrow{\mathrm{H}}=(\overrightarrow{\mathrm{H}} \cdot \nabla) \overrightarrow{\mathrm{q}}+\gamma_{\mathrm{m}} \nabla^{2} \overrightarrow{\mathrm{H}}$

where $\vec{q}$ is the velocity, $\vec{\omega}$ is the spin, $T$ is the temperature, $\vec{H}$ is the magnetic field, $P=p+\frac{\mu_{m}}{2} H_{0}^{2}$ is the hydromagnetic pressure, $\rho$ is the density, $\rho_{0}$ is the density of the fluid at a reference temperature $T=T_{0}, \gamma$ is the acceleration due to gravity, $\zeta$ is the coupling viscosity coefficient or vortex viscosity, $\eta$ is the shear kinematic viscosity coefficient, $l$ is the moment of inertia, $\lambda^{\prime}$ and $\eta^{\prime}$ are the bulk and shear spin viscosity coefficient, $\beta$ is the micropolar heat conduction coefficient, $C_{y}$ is the specific heat, $\chi$ is the thermal conductivity, $\alpha$ is the coefficient of thermal expansion and $\gamma_{m}=1 / \mu_{m} \sigma_{m}$ is the magnetic viscosity $\left(\sigma_{m}\right.$ : electrical conductivity and $\mu_{m}$ : magnetic permeability). All the viscosity coefficients, heat conduction coefficient and thermal conductivity are thermodynamically restricted to be positive quantities on the assumption of Clausius-Duhem inequality (see Eringen [7]). In the energy equation (4) the viscous heating and Joule heating are neglected.

Equations (1)-(6) are solved subject to various velocity and temperature boundary conditions. Further, the no-spin boundary condition is assumed for microrotation. 


\subsection{Reference Steady-State}

In the reference steady-state the fluid is at rest, the temperature distribution across the layer is non-linear and the lines of force of the magnetic field are vertical. This state is described by :

$$
\overrightarrow{\mathrm{q}}_{\mathrm{b}}=0, \vec{\omega}_{\mathrm{b}}=0, \overrightarrow{\mathrm{H}}_{\mathrm{b}}=\mathrm{H}_{0} \hat{k}, p=\mathrm{p}_{\mathrm{b}}(\mathrm{z}), \rho=\rho_{\mathrm{b}}(\mathrm{z}),-\frac{\mathrm{d}}{\Delta \mathrm{T}} \frac{\mathrm{dT} \mathrm{T}_{\mathrm{b}}}{\mathrm{dz}}=\mathrm{f}(\mathrm{z})
$$

The monotonic, non-dimensional basic temperature gradient $f(z)$ which is nonnegative satisfies the condition $\int_{0}^{1} f(z) d z=1$. The non-uniformity in $T_{b}$ as in (7) finds its origin in transient heating or cooling at the boundaries (Siddheshwar and Pranesh $[21,22]$ and references therein). In this paper various reference steadystate temperature gradients are considered and these are defined below:

Table 1:

\begin{tabular}{|c|c|c|}
\hline Model & $\begin{array}{l}\text { Reference steady-state } \\
\text { temperature gradient }\end{array}$ & $f(z)$ \\
\hline 1 & Linear & 1 \\
\hline 2 & Heating from below & $\begin{cases}\varepsilon^{-1} & 0 \leq z<\varepsilon \\
0 & \varepsilon<z \leq 1\end{cases}$ \\
\hline 3 & Cooling from above & $\begin{cases}0 & 0 \leq z<1-\varepsilon \\
\varepsilon^{-1} & 1-\varepsilon<z \leq 1\end{cases}$ \\
\hline 4 & Step function & $\delta(z-\varepsilon)$ \\
\hline 5 & Inverted parabolic & $2(1-z)$ \\
\hline 6 & Parabolic & $2 z$ \\
\hline
\end{tabular}




\subsection{Linear Stability Analysis}

Let the reference steady-state be disturbed by an infinitesimal perturbation in velocity, microrotation, pressure, density, temperature and magnetic field. We now have

$$
\begin{aligned}
& \overrightarrow{\mathrm{q}}=\overrightarrow{\mathrm{q}}_{\mathrm{b}}+\overrightarrow{\mathrm{q}}^{\prime}, \quad \vec{\omega}=\vec{\omega}_{\mathrm{b}}+\vec{\omega}^{\prime}, \quad P=\mathrm{P}_{\mathrm{b}}+\mathrm{P}^{\prime}, \quad \rho=\rho_{\mathrm{b}}+\rho^{\prime}, \\
& \mathrm{T}=\mathrm{T}_{\mathrm{b}}+\mathrm{T}^{\prime}, \quad \overrightarrow{\mathrm{H}}=\overrightarrow{\mathrm{H}}_{\mathrm{b}}+\overrightarrow{\mathrm{H}}^{\prime} .
\end{aligned}
$$

The primes indicate that the quantities are infinitesimal perturbations and subscript $b$ indicates the reference steady-state value. In the present problem we assume the principle of exchange of stability to be valid and hence deal with only stationary convection.

Substituting equation (8) into equations (1)-(6), we get the linearised equations governing the infinitesimal perturbations in the form :

$$
\begin{aligned}
& \nabla \cdot \overrightarrow{\mathrm{q}}^{\prime}=0, \\
& -\nabla \mathrm{P}^{\prime}-\rho^{\prime} \mathrm{gk}+(2 \zeta+\eta) \nabla^{2} \overrightarrow{\mathrm{q}}^{\prime}+\zeta \nabla \times \vec{\omega}^{\prime}+\mu_{\mathrm{m}} \mathrm{H}_{0} \frac{\partial \mathrm{H}_{z}^{\prime}}{\partial \mathrm{z}} \hat{\mathrm{k}}=0, \\
& \left(\lambda^{\prime}+\eta^{\prime}\right) \nabla\left(\nabla \cdot \vec{\omega}^{\prime}\right)+\eta^{\prime} \nabla^{2} \vec{\omega}^{\prime}+\zeta\left(\nabla \times \overrightarrow{\mathrm{q}}^{\prime}-2 \vec{\omega}^{\prime}\right)=0, \\
& -\frac{\Delta \mathrm{T}}{\mathrm{d}} \mathrm{f}(\mathrm{z})\left(\mathrm{W}-\frac{\beta}{\rho_{0} \mathrm{C}_{\mathrm{v}}} \nabla \times \vec{\omega}^{\prime} \hat{\mathrm{k}}\right)=\chi \nabla^{2} \mathrm{~T}^{\prime}, \\
& \rho^{\prime}=-\alpha \rho_{0} \mathrm{~T}^{\prime}, \\
& \mathrm{H}_{0} \frac{\partial \mathrm{w}}{\partial z} \hat{\mathrm{k}}+\gamma_{\mathrm{m}} \nabla^{2} \overrightarrow{\mathrm{H}}^{\prime}=0 .
\end{aligned}
$$

The perturbation equations (9)-(14) are non-dimensionalised using the following definition :

$$
\left(\mathrm{x}^{*}, \mathrm{y}^{*}, \mathrm{z}^{*}\right)=\frac{(\mathrm{x}, \mathrm{y}, \mathrm{z})}{\mathrm{d}}, \overrightarrow{\mathrm{q}}^{*}=\frac{\overrightarrow{\mathrm{q}}^{\prime}}{\chi / \mathrm{d}}, \vec{\omega}^{*}=\frac{\vec{\omega}^{\prime}}{\chi / \mathrm{d}^{2}}, \mathrm{~T}^{*}=\frac{\mathrm{T}^{\prime}}{\Delta \mathrm{T}}, \quad \overrightarrow{\mathrm{H}}^{*}=\frac{\overrightarrow{\mathrm{H}}^{\prime}}{\mathrm{H}_{0}} .
$$

Using equation (13) in equation (10), operating curl twice on the resulting equation, operating curl on equation (11) and non-dimensionalising the two resulting equations and also equations (12) and (14), we get

$$
\left(1+N_{1}\right) \nabla^{4} W+N_{1} \nabla^{2} \Omega_{z}+R\left(\frac{\partial^{2} T}{\partial x^{2}}+\frac{\partial^{2} T}{\partial y^{2}}\right)+Q \frac{\operatorname{Pr}}{P m} \nabla^{2}\left(\frac{\partial H_{z}}{\partial z}\right)=0
$$




$$
\begin{aligned}
& \mathrm{N}_{3} \nabla^{2} \Omega_{\mathrm{z}}-2 \mathrm{~N}_{1} \Omega_{\mathrm{z}}-\mathrm{N}_{1} \nabla^{2} \mathrm{~W}=0, \\
& \nabla^{2} \mathrm{~T}+\mathrm{W}-\mathrm{N}_{5} \Omega_{\mathrm{z}}=0, \\
& \nabla^{2} \mathrm{H}_{\mathrm{z}}+\frac{\operatorname{Pm}}{\operatorname{Pr}} \frac{\partial \mathrm{W}}{\partial \mathrm{z}}=0,
\end{aligned}
$$

where the asterisks have been dropped for simplicity and

$$
\begin{array}{ll}
\mathrm{N}_{1}=\frac{\zeta}{\zeta+\eta} & \text { (Coupling Parameter) } \\
\mathrm{N}_{3}=\frac{\eta^{\prime}}{(\zeta+\eta) \mathrm{d}^{2}} & \text { (Couple Stress Parameter), } \\
\mathrm{N}_{5}=\frac{\beta}{\rho_{0} \mathrm{C}_{\mathrm{v}} \mathrm{d}^{2}} & \text { (Micropolar Heat Conduction Parameter) }
\end{array}
$$$$
\operatorname{Pr}=\frac{\zeta+\eta}{\chi} \quad \text { (Prandtl number) }
$$$$
\mathrm{Pm}=\frac{\zeta+\eta}{\gamma_{\mathrm{m}}} \quad \text { (Magnetic Prandtl number), }
$$$$
\mathrm{R}=\frac{\alpha \mathrm{g} \Delta \mathrm{Td}^{3} \mathrm{p}_{0}}{(\zeta+\eta) \chi} \quad \text { (Rayleigh number) and }
$$

$$
\mathrm{Q}=\frac{\mu_{\mathrm{m}} \mathrm{H}_{0}^{2} \mathrm{~d}^{2}}{(\zeta+\eta) \gamma_{\mathrm{m}}} \quad \text { (Chandrasekhar number) }
$$

The infinitesimal perturbations $W, \Omega, T$ and $H_{z}$ are assumed to be periodic and hence these permit a normal mode solution in the form

$$
\left[\mathrm{W}, \Omega, \mathrm{T}, \mathrm{H}_{\mathrm{z}}\right]=\left[\mathrm{W}(\mathrm{z}), \mathrm{G}(\mathrm{z}), \mathrm{T}(\mathrm{z}), \mathrm{H}_{\mathrm{z}}(\mathrm{z})\right] \exp [\mathrm{i}(\mathrm{lx}+\mathrm{my})] \text {, }
$$

where $l$ and $m$ are horizontal components of the wave number $\vec{a}$. 
Substituting equation (20) into equations (16)-(19), we get

$$
\begin{aligned}
& \left(1+N_{1}\right)\left(D^{2}-a^{2}\right)^{2} W+N_{1}\left(D^{2}-a^{2}\right) G-R a^{2} T+Q \frac{P r}{P m}\left(D^{2}-a^{2}\right) D H_{z}=0, \\
& N_{1}\left(D^{2}-a^{2}\right) W-N_{3}\left(D^{2}-a^{2}\right) G+2 N_{1} G=0 \\
& \left(D^{2}-a^{2}\right) T+f(z)\left(W-N_{5} G\right)=0 \\
& \left(D^{2}-a^{2}\right) H_{z}+\frac{P m}{P r} D W=0
\end{aligned}
$$

where $\mathrm{D} \equiv \frac{\mathrm{d}}{\mathrm{dz}}$.

The sets of ordinary differential equations (21)-(24) are approximations based on physical considerations to the system of partial differential equations (16)-(19). Although the relationship between the solutions of the governing partial differential equations and the corresponding ordinary differential equations has not been established, these linear models reproduce qualitatively the convective phenomena observable through the full system.

Eliminating $\mathrm{H}_{2}$ between equations (21) and (24), we get

$$
\left(1+N_{1}\right)\left(D^{2}-a^{2}\right)^{2} W+N_{1}\left(D^{2}-a^{2}\right) G-R a^{2} T-Q D^{2} W=0 .
$$

Equations (22), (23) and (25) are solved subject to the following boundary conditions:

$W=D W=T=G=0$ at $z=0, W=D^{2} W=D T=G=0$ at $z=1$,

Equation (26) indicates the use of rigid, isothermal lower boundary and upper, free, thermally insulating boundary (with respect to the perturbation). The condition on $G$ is the spin-vanishing boundary condition.

We now use the single-term Galerkin method to find the critical eigenvalue to equations (22), (23) and (25) that gives general results on the eigen value of the problem for various basic temperature gradients using simple, polynomial, trial functions for the lowest eigen value. We obtain an approximate solution of the differential equations with the given boundary conditions by choosing trial functions for velocity, microrotation and temperature perturbations that may satisfy some of the boundary conditions but may not exactly satisfy the differential equations. This 
leads to residuals when the trial functions are substituted into the differential equations. The Galerkin method requires the residual to be orthogonal to each individual trial function.

In the Galerkin procedure, we expand the velocity, microrotation and temperature by,

$$
w(z, t)=\sum A_{j}(t) W_{i}(z), \quad G(z, t)=\sum B_{j}(t) G_{i}(z), \quad T(z, t)=\sum C_{j}(t) T_{i}(z)
$$

where $W_{i}(z), G_{i}(z)$ and $T_{i}(z)$ are polynomials in $z$ that generally have to satisfy the given boundary conditions. For the single term Galerkin expansion technique we take $\mathrm{i}=\mathrm{i}=\mathrm{l}$.

Multiplying equation (25) by W, equation (22) by $G$ and equation (23) by T, integrating the resulting equation by parts with respect to $z$ from 0 to $l$ and taking $W=A W_{1}$, $G=B G_{1}$ and $T=C T_{1}$ in which $A, B$ and $C$ are constants and $W_{1}, G_{1}$ and $T_{1}$ are trial functions. This procedure yields the following equation for the Rayleigh number $R$ :

$$
R=\frac{\left\langle T_{1}\left(D^{2}-a^{2}\right) T_{1}\right\rangle\left[C_{1} C_{2}+N_{1}^{2} C_{3}\right]}{a^{2}\left\langle W_{1} T_{1}\right\rangle C_{4}}
$$

where

$$
\begin{aligned}
& C_{1}=N_{3}\left\langle G_{1}\left(D^{2}-a^{2}\right) G_{1}\right\rangle-2 N_{1}\left\langle G_{1}^{2}\right\rangle, \\
& C_{2}=\left(1+N_{1}\right)\left\langle W_{1}\left(D^{2}-a^{2}\right)^{2} W_{1}\right\rangle-Q\left\langle W_{1} D^{2} W_{1}\right\rangle, \\
& C_{3}=\left\langle W_{1}\left(D^{2}-a^{2}\right) G_{1}\right\rangle\left\langle G_{1}\left(D^{2}-a^{2}\right) W_{1}\right\rangle, \\
& C_{4}=N_{1} N_{5}\left\langle f(z) G_{1} T_{1}\right\rangle\left\langle G_{1}\left(D^{2}-a^{2}\right) W_{1}\right\rangle-\left\langle f(z) W_{1} T_{1}\right\rangle C_{1}
\end{aligned}
$$

In equation (27), $\langle--\rangle$ denotes integration with respect to $z$ between $z=0$ and $z=1$. We note here that $R$ in equation (27) is a functional and the Euler-Lagrange equations for the extremisation of $R$ are equations $(22),(23)$ and (25). 
The value of critical Rayleigh number depends on the boundaries. In this paper we consider the following boundary combinations:

(a) Free-free isothermal / adiabatic, no spin.

(b) Rigid-rigid isothermal / adiabatic, no spin.

(c) Rigid-free isothermal / adiabatic, no spin.

(d) Lower rigid isothermal and upper free adiabatic, no spin.

\section{Critical Rayleigh number for free-free, isothermal, no spin:}

Table (2): Isothermal: $N_{1}=0.1, N_{3}=2.0, N_{5}=1.0, Q=10.0$

\begin{tabular}{|c|c|c|c|c|c|c|}
\hline Boundary & \multicolumn{2}{|c|}{ Free - Free } & \multicolumn{2}{|c|}{ Rigid - Free } & \multicolumn{2}{|c|}{ Rigid - Rigid } \\
\hline Profiles & $\varepsilon_{\mathrm{c}}$ & Ratio of $R_{c i}$ & $\varepsilon_{c}$ & Ratio of $R_{\mathrm{ci}}$ & $\varepsilon_{\mathrm{c}}$ & Ratio of $\mathbf{R}_{\mathrm{ci}}$ \\
\hline Heating from below & 0.72 & $\mathrm{R}_{\mathrm{c} 2}=\mathrm{R}_{\mathrm{cl}} / 1.2070$ & 0.76 & $\mathrm{R}_{\mathrm{c} 2}=\mathrm{R}_{\mathrm{c} 1} / 1.1682$ & 0.70 & $\mathrm{R}_{\mathrm{c} 2}=\mathrm{R}_{\mathrm{cl}} / 1.2513$ \\
\hline Cooling from obove & 0.72 & $\mathrm{R}_{\mathrm{c} 3}=\mathrm{R}_{\mathrm{cl}} / 1.2070$ & 0.65 & $\mathrm{R}_{\mathrm{c} 3}=\mathrm{R}_{\mathrm{cl}} / 1.3392$ & 0.70 & $\mathrm{R}_{\mathrm{c} 3}=\mathrm{R}_{\mathrm{cl}} / 1.2513$ \\
\hline Step function & 0.52 & $\mathrm{R}_{\mathrm{c} 4}=\mathrm{R}_{\mathrm{cl}} / 1.2070$ & 0.54 & $\mathrm{R}_{\mathrm{c} 4}=\mathrm{R}_{\mathrm{cl}} / 2.0798$ & 0.50 & $\mathrm{R}_{\mathrm{c} 4}=\mathrm{R}_{\mathrm{c} 1} / 2.2033$ \\
\hline Inverted porobolic & - & $\mathrm{R}_{\mathrm{cs}}=\mathrm{R}_{\mathrm{cl}}$ & - & $\mathrm{R}_{\mathrm{c} 4}=\mathrm{R}_{\mathrm{c}} / 0.9191$ & - & $\mathrm{R}_{\mathrm{cs}}=\mathrm{R}_{\mathrm{cl}}$ \\
\hline Parabolic & - & $\mathrm{R}_{\mathrm{c} 6}=\mathrm{R}_{\mathrm{cl}}$ & - & $\mathrm{R}_{\mathrm{c} 4}=\mathrm{R}_{\mathrm{cl}} / 1.0808$ & - & $\mathrm{R}_{\mathrm{c} 6}=\mathrm{R}_{\mathrm{cl}}$ \\
\hline
\end{tabular}

The boundary conditions are

$\mathrm{W}=\mathrm{D}^{2} \mathrm{~W}=\mathrm{T}=\mathrm{G}=0, \quad$ at $\quad \mathrm{z}=0,1$.

The trial functions satisfying (28) are

$$
\mathrm{W}_{1}=\mathrm{z}^{4}-2 \mathrm{z}^{3}+z, \quad \mathrm{~T}_{1}=\mathrm{z}(1-\mathrm{z}), \quad \mathrm{G}_{1}=\mathrm{z}(1-\mathrm{z})
$$


Substituting (29) in equation (27) and performing the integration, we get

$$
R=\frac{\left(10+a^{2}\right)}{1530 a^{2}}\left\{\frac{28 y_{1}\left[\left(1+N_{1}\right) y_{2}+306 Q\right]-3 N_{1}^{2} y_{3}^{2}}{14 y_{1}\left\langle f(z) W_{1} T_{1}\right\rangle-N_{1} N_{5} y_{3}\left\langle f(z) T_{1} G_{1}\right\rangle}\right\},
$$

where

$$
\begin{aligned}
& y_{1}=N_{3}\left(10+a^{2}\right)+2 N_{1}, \\
& y_{2}=3024+612 a^{2}+31 a^{4}, \\
& y_{3}=168+17 a^{2} .
\end{aligned}
$$

For a given $f(z), R$ attains its minimum value $R_{c}$ at $a=a_{c}$.

The integrals $\left\langle\mathrm{f}(\mathrm{z}) \mathrm{W}_{1} \mathrm{~T}_{1}\right\rangle$ and $\left\langle\mathrm{f}(\mathrm{z}) \mathrm{T}_{1} \mathrm{G}_{1}\right\rangle$ will have the following forms depending on the nature of basic temperature profile.

Model 1. Linear temperature profile:

$$
\left\langle\mathrm{f}(\mathrm{z}) \mathrm{W}_{1} \mathrm{~T}_{1}\right\rangle=\frac{17}{420}, \quad\left\langle\mathrm{f}(\mathrm{z}) \mathrm{T}_{1} \mathrm{G}_{1}\right\rangle=\frac{1}{30} .
$$

Model 2. Piecewise linear heating from below temperature profile:

$$
\begin{aligned}
& \left\langle\mathrm{f}(\mathrm{z}) \mathrm{W}_{\mathrm{1}} \mathrm{T}_{1}\right\rangle=\frac{1}{420}\left(-60 \varepsilon^{6}+210 \varepsilon^{5}-168 \varepsilon^{4}-105 \varepsilon^{3}+140 \varepsilon^{2}\right), \\
& \left\langle\mathrm{f}(\mathrm{z}) \mathrm{T}_{1} \mathrm{G}_{1}\right\rangle=\frac{1}{30}\left(6 \varepsilon^{4}-15 \varepsilon^{3}+10 \varepsilon^{2}\right) .
\end{aligned}
$$

Model 3. Piecewise linear cooling from above temperature profile:

$$
\begin{aligned}
& \left.\left\langle\mathrm{f}(\mathrm{z}) \mathrm{W}_{1} \mathrm{~T}_{1}\right\rangle=\frac{1}{420}\left(-60 \varepsilon^{6}+210 \varepsilon^{5}-168 \varepsilon^{4}-105 \varepsilon^{3}+140 \varepsilon^{2}\right),\right\} . \\
& \left\langle\mathrm{f}(\mathrm{z}) \mathrm{T}_{1} \mathrm{G}_{1}\right\rangle=\frac{1}{30}\left(6 \varepsilon^{4}-15 \varepsilon^{3}+10 \varepsilon^{2}\right) .
\end{aligned}
$$

Model 4. Step function temperature profile:

$$
\begin{aligned}
& \left\langle\mathrm{f}(\mathrm{z}) \mathrm{W}_{1} \mathrm{~T}_{1}\right\rangle=-\varepsilon^{6}+3 \varepsilon^{5}-2 \varepsilon^{4}-\varepsilon^{3}+\varepsilon^{2}, \\
& \left\langle\mathrm{f}(\mathrm{z}) \mathrm{T}_{1} \mathrm{G}_{1}\right\rangle=\varepsilon^{4}-2 \varepsilon^{3}+\varepsilon^{2} .
\end{aligned}
$$


Model 5. Inverted temperature profile:

$$
\left\langle\mathrm{f}(\mathrm{z}) \mathrm{W}_{1} \mathrm{~T}_{1}\right\rangle=\frac{17}{420}, \quad\left\langle\mathrm{f}(\mathrm{z}) \mathrm{T}_{1} \mathrm{G}_{1}\right\rangle=\frac{1}{30}
$$

Model 6. Parabolic temperature profile:

$$
\left\langle\mathrm{f}(\mathrm{z}) \mathrm{W}_{1} \mathrm{~T}_{1}\right\rangle=\frac{17}{420}, \quad\left\langle\mathrm{f}(\mathrm{z}) \mathrm{T}_{1} \mathrm{G}_{1}\right\rangle=\frac{1}{30}
$$

\section{Critical Rayleigh number for rigid-rigid, isothermal, no spin:}

The boundary conditions are

$$
\mathrm{W}=\mathrm{DW}=\mathrm{T}=\mathrm{G}=0 \text {, at } \mathrm{z}=0,1 \text {. }
$$

The trial functions satisfying (31) are

$$
\mathrm{W}_{1}=\mathrm{z}^{4}-2 \mathrm{z}^{3}+\mathrm{z}^{2}, \quad \mathrm{~T}_{1}=\mathrm{z}(1-\mathrm{z}), \quad \mathrm{G}_{1}=\mathrm{z}(1-\mathrm{z})
$$

Substituting (32) in equation (27) and performing the integration, we get

$$
R=\frac{\left(10+a^{2}\right)}{270 a^{2}}\left\{\frac{28 y_{1}\left[\left(1+N_{1}\right) y_{2}+12 Q\right]-3 N_{1}^{2} y_{3}^{2}}{14 y_{1}\left\langle f(z) W_{1} T_{1}\right\rangle-N_{1} N_{5} y_{3}\left\langle f(z) T_{1} G_{1}\right\rangle}\right\},
$$

where

$$
\begin{aligned}
& y_{1}=N_{3}\left(10+a^{2}\right)+2 N_{1}, \\
& y_{2}=504+24 a^{2}+a^{4}, \\
& y_{3}=28+3 a^{2} .
\end{aligned}
$$

For a given $f(z), R$ attains its minimum value $R_{c}$ at $a=a_{c}$.

The integrals $\left\langle f(z) W_{1} T_{1}\right\rangle$ and $\left\langle f(z) T_{1} G_{1}\right\rangle$ will have the following forms depending on the nature of basic temperature profile. 
Model 1. Linear temperature profile:

$\left\langle\mathrm{f}(\mathrm{z}) \mathrm{W}_{1} \mathrm{~T}_{1}\right\rangle=\frac{1}{140}, \quad\left\langle\mathrm{f}(\mathrm{z}) \mathrm{T}_{1} \mathrm{G}_{1}\right\rangle=\frac{1}{30}$.

Model 2. Piecewise linear heating from below temperature profile:

$$
\begin{aligned}
& \left\langle\mathrm{f}(\mathrm{z}) \mathrm{W}_{1} \mathrm{~T}_{1}\right\rangle=\frac{1}{140}\left(-20 \varepsilon^{6}+70 \varepsilon^{5}-84 \varepsilon^{4}+35 \varepsilon^{3}\right), \\
& \left\langle\mathrm{f}(\mathrm{z}) \mathrm{T}_{1} \mathrm{G}_{1}\right\rangle=\frac{1}{30}\left(6 \varepsilon^{4}-15 \varepsilon^{3}+10 \varepsilon^{2}\right) .
\end{aligned}
$$

Model 3. Piecewise linear cooling from above temperature profile:

$$
\begin{aligned}
& \left\langle\mathrm{f}(\mathrm{z}) \mathrm{W}_{1} \mathrm{~T}_{1}\right\rangle=\frac{1}{140}\left(-20 \varepsilon^{6}+70 \varepsilon^{5}-84 \varepsilon^{4}+35 \varepsilon^{3}\right), \\
& \left\langle\mathrm{f}(\mathrm{z}) \mathrm{T}_{1} \mathrm{G}_{1}\right\rangle=\frac{1}{30}\left(6 \varepsilon^{4}-15 \varepsilon^{3}+10 \varepsilon^{2}\right) .
\end{aligned}
$$

Model 4. Step function temperature profile:

$$
\begin{aligned}
& \left\langle\mathrm{f}(\mathrm{z}) \mathrm{W}_{1} \mathrm{~T}_{1}\right\rangle=-\varepsilon^{6}+3 \varepsilon^{5}-3 \varepsilon^{4}+\varepsilon^{3}, \\
& \left\langle\mathrm{f}(\mathrm{z}) \mathrm{T}_{1} \mathrm{G}_{\mathrm{l}}\right\rangle=\varepsilon^{4}-2 \varepsilon^{3}+\varepsilon^{2} .
\end{aligned}
$$

Model 5. Inverted temperature profile:

$$
\left\langle\mathrm{f}(\mathrm{z}) \mathrm{W}_{1} \mathrm{~T}_{1}\right\rangle=\frac{1}{140}, \quad\left\langle\mathrm{f}(\mathrm{z}) \mathrm{T}_{1} \mathrm{G}_{1}\right\rangle=\frac{1}{30} .
$$

Model 6. Parabolic temperature profile:

$$
\left\langle\mathrm{f}(\mathrm{z}) \mathrm{W}_{1} \mathrm{~T}_{1}\right\rangle=\frac{1}{140}, \quad\left\langle\mathrm{f}(\mathrm{z}) \mathrm{T}_{1} \mathrm{G}_{1}\right\rangle=\frac{1}{30} .
$$




\section{Critical Rayleigh number for lower rigid and upper free isothermal, no spin:}

The boundary conditions are

$$
\left.\begin{array}{lll}
\mathrm{W}=\mathrm{DW}=\mathrm{T}=\mathrm{G}=0 & \text { at } & \mathrm{z}=0 \\
\mathrm{~W}=\mathrm{D}^{2} \mathrm{~W}=\mathrm{T}=\mathrm{G}=0 & \text { at } & \mathrm{z}=1
\end{array}\right\},
$$

The trial functions satisfying (34) are

$$
\mathrm{W}_{1}=2 \mathrm{z}^{4}-5 \mathrm{z}^{3}+3 \mathrm{z}^{2}, \quad \mathrm{~T}_{1}=\mathrm{z}(1-\mathrm{z}), \quad \mathrm{G}_{1}=\mathrm{z}(1-\mathrm{z})
$$

Substituting (35) in equation (27) and performing the integration, we get

$$
R=\frac{\left(10+a^{2}\right)}{1170 a^{2}}\left\{\frac{28 y_{1}\left[\left(1+N_{1}\right) y_{2}+216 Q\right]-3 N_{1}^{2} y_{3}^{2}}{28 y_{1}\left\langle f(z) W_{1} T_{1}\right\rangle-N_{1} N_{5} y_{3}\left\langle f(z) T_{1} G_{1}\right\rangle}\right\}
$$

where

$$
\begin{aligned}
& y_{1}=N_{3}\left(10+a^{2}\right)+2 N_{1}, \\
& y_{2}=4536+432 a^{2}+19 a^{4}, \\
& y_{3}=126+13 a^{2} .
\end{aligned}
$$

For a given $f(z), R$ attains its minimum value $R_{c}$ at $a=a_{c}$.

The integrals $\left\langle\mathrm{f}(\mathrm{z}) \mathrm{W}_{1} \mathrm{~T}_{1}\right\rangle$ and $\left\langle\mathrm{f}(\mathrm{z}) \mathrm{T}_{1} \mathrm{G}_{1}\right\rangle$ will have the following forms depending on the nature of basic temperature profile.

Model 1. Linear temperature profile:

$\left\langle\mathrm{f}(\mathrm{z}) \mathrm{W}_{1} \mathrm{~T}_{1}\right\rangle=\frac{13}{840}, \quad\left\langle\mathrm{f}(\mathrm{z}) \mathrm{T}_{1} \mathrm{G}_{1}\right\rangle=\frac{1}{30}$. 
Model 2. Piecewise linear heating from below temperature profile:

$$
\begin{aligned}
& \left\langle\mathrm{f}(\mathrm{z}) \mathrm{W}_{1} \mathrm{~T}_{1}\right\rangle=\frac{1}{840}\left(-120 \varepsilon^{6}+490 \varepsilon^{5}-672 \varepsilon^{4}+315 \varepsilon^{3}\right), \\
& \left\langle\mathrm{f}(\mathrm{z}) \mathrm{T}_{1} \mathrm{G}_{1}\right\rangle=\frac{1}{30}\left(6 \varepsilon^{4}-15 \varepsilon^{3}+10 \varepsilon^{2}\right) .
\end{aligned}
$$

Model 3. Piecewise linear cooling from above temperature profile:

$$
\begin{aligned}
& \left\langle\mathrm{f}(\mathrm{z}) \mathrm{W}_{1} \mathrm{~T}_{1}\right\rangle=\frac{1}{840}\left(-120 \varepsilon^{6}+350 \varepsilon^{5}-252 \varepsilon^{4}-105 \varepsilon^{3}+140 \varepsilon^{2}\right), \\
& \left\langle\mathrm{f}(\mathrm{z}) \mathrm{T}_{1} \mathrm{G}_{1}\right\rangle=\frac{1}{30}\left(6 \varepsilon^{4}-15 \varepsilon^{3}+10 \varepsilon^{2}\right) .
\end{aligned}
$$

Model 4. Step function temperature profile:

$$
\begin{aligned}
& \left\langle\mathrm{f}(\mathrm{z}) \mathrm{W}_{1} \mathrm{~T}_{1}\right\rangle=-\varepsilon^{6}+\frac{7}{2} \varepsilon^{5}-4 \varepsilon^{4}+\frac{3}{2} \varepsilon^{3}, \\
& \left\langle\mathrm{f}(\mathrm{z}) \mathrm{T}_{1} \mathrm{G}_{1}\right\rangle=\varepsilon^{4}-2 \varepsilon^{3}+\varepsilon^{2} .
\end{aligned}
$$

Model 5. Inverted temperature profile:

$$
\left\langle\mathrm{f}(\mathrm{z}) \mathrm{W}_{1} \mathrm{~T}_{1}\right\rangle=\frac{1}{70}, \quad\left\langle\mathrm{f}(\mathrm{z}) \mathrm{T}_{1} \mathrm{G}_{1}\right\rangle=\frac{1}{30} .
$$

Model 6. Parabolic temperature profile:

$$
\left\langle\mathrm{f}(\mathrm{z}) \mathrm{W}_{1} \mathrm{~T}_{1}\right\rangle=\frac{1}{60}, \quad\left\langle\mathrm{f}(\mathrm{z}) \mathrm{T}_{1} \mathrm{G}_{1}\right\rangle=\frac{1}{30} .
$$




\section{Critical Rayleigh number for free-free adiabatic, no spin:}

Table (3): Adiabatic: $N_{1}=0.1, N_{3}=2.0, N_{5}=1.0, Q=10.0$

\begin{tabular}{|l|c|c|c|c|c|c|}
\hline Boundary & \multicolumn{2}{|c|}{ Free - Free } & \multicolumn{2}{c|}{ Rigid - Free } & \multicolumn{2}{c|}{ Rigid - Rigid } \\
\hline Profiles & $\varepsilon_{\mathrm{c}}$ & Ratio of $\mathrm{R}_{\mathrm{ci}}$ & $\varepsilon_{\mathrm{c}}$ & Ratio of $\mathrm{R}_{\mathrm{ci}}$ & $\varepsilon_{\mathrm{c}}$ & \multicolumn{2}{c|}{ Ratio of $\mathrm{R}_{\mathrm{ci}}$} \\
\hline Heating from below & 0.75 & $\mathrm{R}_{\mathrm{c} 2}=\mathrm{R}_{\mathrm{cl}} / 1.1373$ & 0.82 & $\mathrm{R}_{\mathrm{c} 2}=\mathrm{R}_{\mathrm{cl}} / 1.1035$ & 0.72 & $\mathrm{R}_{\mathrm{c} 2}=\mathrm{R}_{\mathrm{cl}} / 1.2015$ \\
\hline Cooling from above & 0.75 & $\mathrm{R}_{\mathrm{c} 3}=\mathrm{R}_{\mathrm{cl}} / 1.1373$ & 0.63 & $\mathrm{R}_{\mathrm{c} 3}=\mathrm{R}_{\mathrm{cl}} / 1.2775$ & 0.72 & $\mathrm{R}_{\mathrm{c} 3}=\mathrm{R}_{\mathrm{cl}} / 1.2015$ \\
\hline Step function & 0.50 & $\mathrm{R}_{\mathrm{c} 4}=\mathrm{R}_{\mathrm{cl}} / 1.5657$ & 0.57 & $\mathrm{R}_{\mathrm{c} 4}=\mathrm{R}_{\mathrm{cl}} / 1.6753$ & 0.50 & $\mathrm{R}_{\mathrm{c} 4}=\mathrm{R}_{\mathrm{cl}} / 1.8945$ \\
\hline Inverted parabolic & - & $\mathrm{R}_{\mathrm{c} 5}=\mathrm{R}_{\mathrm{cl}}$ & - & $\mathrm{R}_{\mathrm{c} 4}=\mathrm{R}_{\mathrm{cl}} / 0.8830$ & - & $\mathrm{R}_{\mathrm{c} 5}=\mathrm{R}_{\mathrm{cl}}$ \\
\hline Parabolic & - & $\mathrm{R}_{\mathrm{c} 6}=\mathrm{R}_{\mathrm{cl}}$ & - & $R_{\mathrm{C} 4}=R_{c l} / 1.1168$ & - & $\mathrm{R}_{\mathrm{c} 6}=\mathrm{R}_{\mathrm{cl}}$ \\
\hline
\end{tabular}

The boundary conditions are

$$
\mathrm{W}=\mathrm{D}^{2} \mathrm{~W}=\mathrm{DT}=\mathrm{G}=0 \text {, at } \mathrm{z}=0,1
$$

The trial functions satisfying (37) are

$$
W_{1}=z^{4}-2 z^{3}+z, \quad T_{1}=1, \quad G_{1}=z(1-z)
$$

Substituting (38) in equation (27) and performing the integration, we get

$$
\mathrm{R}=\frac{1}{252}\left\{\frac{28 \mathrm{y}_{1}\left[\left(1+\mathrm{N}_{1}\right) \mathrm{y}_{2}+306 \mathrm{Q}\right]-3 \mathrm{~N}_{1}^{2} \mathrm{y}_{3}^{2}}{14 \mathrm{y}_{1}\left\langle\mathrm{f}(\mathrm{z}) \mathrm{W}_{1} \mathrm{~T}_{1}\right\rangle-\mathrm{N}_{1} \mathrm{~N}_{5} \mathrm{y}_{3}\left\langle\mathrm{f}(\mathrm{z}) \mathrm{T}_{1} \mathrm{G}_{1}\right\rangle}\right\}
$$


where

$$
\begin{aligned}
& y_{1}=N_{3}\left(10+a^{2}\right)+2 N_{1}, \\
& y_{2}=3024+612 a^{2}+31 a^{4}, \\
& y_{3}=168+17 a^{2} .
\end{aligned}
$$

For a given $f(z), R$ attains its minimum value $R_{c}$ at $a=a_{c}$.

The integrals $\left\langle f(z) W_{1} T_{1}\right\rangle$ and $\left\langle f(z) T_{1} G_{1}\right\rangle$ will have the following forms depending on the nature of basic temperature profile.

Model 1. Linear temperature profile:

$$
\left\langle\mathrm{f}(\mathrm{z}) \mathrm{W}_{1} \mathrm{~T}_{1}\right\rangle=\frac{1}{5}, \quad\left\langle\mathrm{f}(\mathrm{z}) \mathrm{T}_{1} \mathrm{G}_{1}\right\rangle=\frac{1}{6} .
$$

Model 2. Piecewise linear heating from below temperature profile:

$$
\begin{aligned}
& \left\langle f(z) W_{1} T_{1}\right\rangle=\frac{1}{10}\left(2 \varepsilon^{4}-5 \varepsilon^{3}+5 \varepsilon\right), \\
& \left\langle f(z) T_{1} G_{1}\right\rangle=\frac{1}{6}\left(-2 \varepsilon^{2}+3 \varepsilon\right) .
\end{aligned}
$$

Model 3. Piecewise linear cooling from above temperature profile:

$$
\begin{aligned}
& \left\langle\mathrm{f}(\mathrm{z}) \mathrm{W}_{1} \mathrm{~T}_{1}\right\rangle=\frac{1}{10}\left(2 \varepsilon^{4}-5 \varepsilon^{3}+5 \varepsilon\right), \\
& \left\langle\mathrm{f}(\mathrm{z}) \mathrm{T}_{1} \mathrm{G}_{1}\right\rangle=\frac{1}{6}\left(-2 \varepsilon^{2}+3 \varepsilon\right) .
\end{aligned}
$$

Model 4. Step function temperature profile:

$$
\begin{aligned}
& \left\langle f(z) W_{1} T_{1}\right\rangle=\varepsilon^{4}-2 \varepsilon^{3}+\varepsilon, \\
& \left\langle f(z) T_{1} G_{1}\right\rangle=-\varepsilon^{2}+\varepsilon .
\end{aligned}
$$


Model 5. Inverted temperature profile:

$\left\langle\mathrm{f}(\mathrm{z}) \mathrm{W}_{1} \mathrm{~T}_{1}\right\rangle=\frac{1}{5}, \quad\left\langle\mathrm{f}(\mathrm{z}) \mathrm{T}_{1} \mathrm{G}_{1}\right\rangle=\frac{1}{6}$.

Model 6. Parabolic temperature profile:

$\left\langle\mathrm{f}(\mathrm{z}) \mathrm{W}_{1} \mathrm{~T}_{1}\right\rangle=\frac{1}{5}, \quad\left\langle\mathrm{f}(\mathrm{z}) \mathrm{T}_{1} \mathrm{G}_{1}\right\rangle=\frac{1}{6}$.

\section{Critical Rayleigh number for rigid-rigid adiabatic, no spin:}

The boundary conditions are

$\mathrm{W}=\mathrm{DW}=\mathrm{DT}=\mathrm{G}=0$, at $\mathrm{z}=0,1$.

The trial functions satisfying $(40)$ are

$\mathrm{W}_{1}=\mathrm{z}^{4}-2 \mathrm{z}^{3}+\mathrm{z}^{2}, \quad \mathrm{~T}_{1}=1, \quad \mathrm{G}_{1}=\mathrm{z}(1-\mathrm{z})$

Substituting (41) in equation (27) and performing the integration, we get

$R=\frac{1}{42}\left\{\frac{28 \mathrm{y}_{1}\left[\left(1+\mathrm{N}_{1}\right) \mathrm{y}_{2}+12 \mathrm{Q}\right]-3 \mathrm{~N}_{1}^{2} \mathrm{y}_{3}^{2}}{14 \mathrm{y}_{1}\left\langle\mathrm{f}(\mathrm{z}) \mathrm{W}_{1} \mathrm{~T}_{1}\right\rangle-\mathrm{N}_{1} \mathrm{~N}_{5} \mathrm{y}_{3}\left\langle f(\mathrm{z}) \mathrm{T}_{1} \mathrm{G}_{1}\right\rangle}\right\}$,

where

$y_{1}=N_{3}\left(10+a^{2}\right)+2 N_{1}$,

$\mathrm{y}_{2}=504+24 \mathrm{a}^{2}+\mathrm{a}^{4}$,

$\mathrm{y}_{3}=28+3 \mathrm{a}^{2}$.

For a given $f(z), R$ attains its minimum value $R_{c}$ at $a=a_{c}$.

The integrals $\left\langle f(z) W_{1} T_{1}\right\rangle$ and $\left\langle f(z) T_{1} G_{1}\right\rangle$ will have the following forms depending on the nature of basic temperature profile. 
Model 1. Linear temperature profile:

$\left\langle\mathrm{f}(\mathrm{z}) \mathrm{W}_{1} \mathrm{~T}_{1}\right\rangle=\frac{1}{30}, \quad\left\langle\mathrm{f}(\mathrm{z}) \mathrm{T}_{1} \mathrm{G}_{1}\right\rangle=\frac{1}{6}$.

Model 2. Piecewise linear heating from below temperature profile:

$$
\begin{aligned}
& \left\langle\mathrm{f}(\mathrm{z}) \mathrm{W}_{1} \mathrm{~T}_{1}\right\rangle=\frac{1}{30}\left(6 \varepsilon^{4}-15 \varepsilon^{3}+10 \varepsilon^{3}\right), \\
& \left\langle\mathrm{f}(\mathrm{z}) \mathrm{T}_{1} \mathrm{G}_{1}\right\rangle=\frac{1}{6}\left(-2 \varepsilon^{2}+3 \varepsilon\right) .
\end{aligned}
$$

Model 3. Piecewise linear cooling from above temperature profile:

$$
\begin{aligned}
& \left\langle\mathrm{f}(\mathrm{z}) \mathrm{W}_{1} \mathrm{~T}_{1}\right\rangle=\frac{1}{30}\left(6 \varepsilon^{4}-15 \varepsilon^{3}+10 \varepsilon^{3}\right), \\
& \left\langle\mathrm{f}(\mathrm{z}) \mathrm{T}_{1} \mathrm{G}_{1}\right\rangle=\frac{1}{6}\left(-2 \varepsilon^{2}+3 \varepsilon\right) .
\end{aligned}
$$

Model 4. Step function temperature profile:

$$
\begin{aligned}
& \left\langle\mathrm{f}(\mathrm{z}) \mathrm{W}_{1} \mathrm{~T}_{1}\right\rangle=\varepsilon^{4}-2 \varepsilon^{3}+\varepsilon^{2}, \\
& \left\langle\mathrm{f}(\mathrm{z}) \mathrm{T}_{1} \mathrm{G}_{1}\right\rangle=-\varepsilon^{2}+\varepsilon .
\end{aligned}
$$

Model 5. Inverted temperature profile:

$$
\left\langle\mathrm{f}(\mathrm{z}) \mathrm{W}_{1} \mathrm{~T}_{1}\right\rangle=\frac{1}{30}, \quad\left\langle\mathrm{f}(\mathrm{z}) \mathrm{T}_{1} \mathrm{G}_{1}\right\rangle=\frac{1}{6} .
$$

Model 6. Parabolic temperature profile:

$$
\left\langle\mathrm{f}(\mathrm{z}) \mathrm{W}_{1} \mathrm{~T}_{\mathrm{l}}\right\rangle=\frac{1}{30}, \quad\left\langle\mathrm{f}(\mathrm{z}) \mathrm{T}_{1} \mathrm{G}_{1}\right\rangle=\frac{1}{6} .
$$




\section{Critical Rayleigh number for lower rigid and upper free adiabatic, no spin :}

Table (4): Lower boundary rigid-isothermal and upper boundary free-adiabatic.

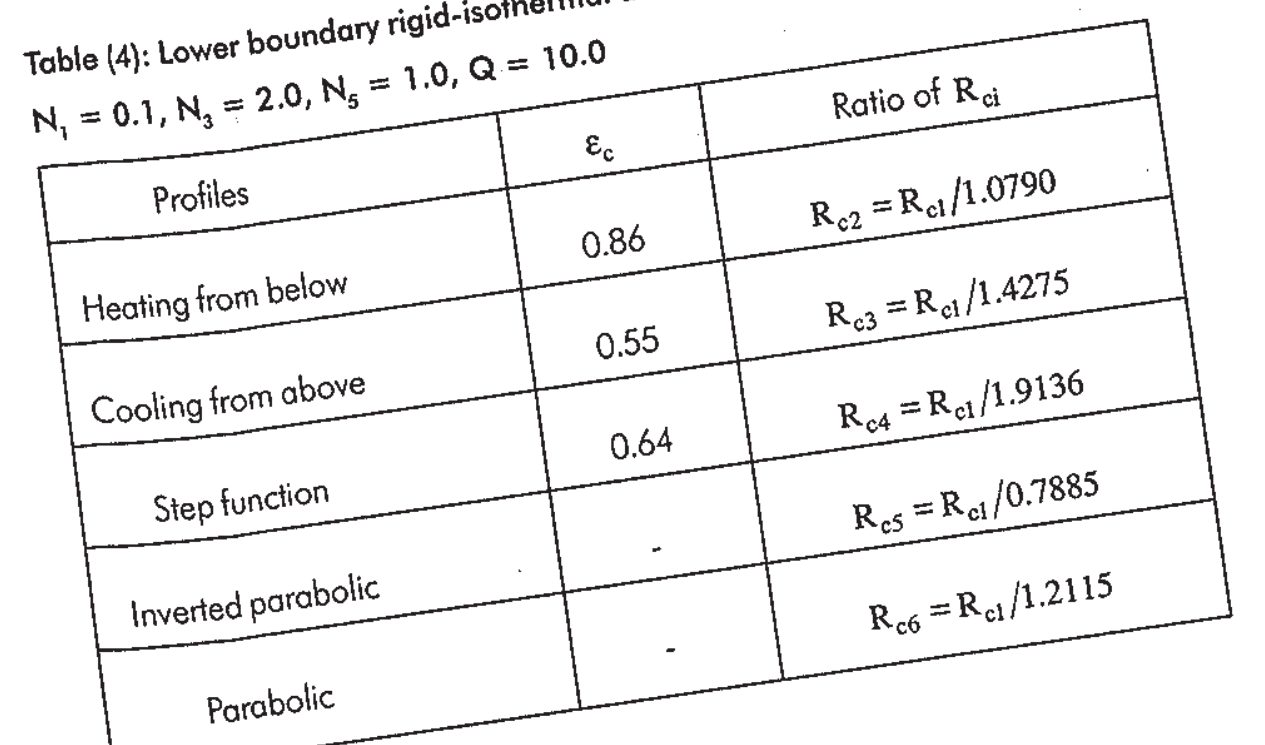

The boundary conditions are

$\left.\begin{array}{l}W=D W=D T=G=0 \quad \text { at } z=0 \\ W=D^{2} W=D T=G=0 \quad \text { at } z=1\end{array}\right\}$

The trial functions satisfying (43) are

$W_{1}=2 z^{4}-5 z^{3}+3 z^{2}, \quad T_{1}=1, \quad G_{1}=z(1-z)$ $R=\frac{1}{189}\left\{\frac{28 y_{1}\left[\left(1+N_{1}\right) y_{2}+216 Q\right]-3 N_{1}^{2} y_{3}^{2}}{28 y_{1}\left\langle f(z) W_{1} T_{1}\right\rangle-N_{1} N_{5} y_{3}\left(f(z) T_{1} G_{1}\right)}\right\}$, 
where

$\mathrm{y}_{1}=\mathrm{N}_{3}\left(10+\mathrm{a}^{2}\right)+2 \mathrm{~N}_{1}$,

$\mathrm{y}_{2}=4536+432 \mathrm{a}^{2}+19 \mathrm{a}^{4}$,

$y_{3}=126+13 a^{2}$.

For a given $f(z), R$ attains its minimum value $R_{c}$ at $a=a_{c}$.

The integrals $\left\langle f(z) W_{1} T_{1}\right\rangle$ and $\left\langle f(z) T_{1} G_{1}\right\rangle$ will have the following forms depending on the nature of basic temperature profile.

Model 1. Linear temperature profile:

$\left\langle\mathrm{f}(\mathrm{z}) \mathrm{W}_{1} \mathrm{~T}_{1}\right\rangle=\frac{3}{40}, \quad\left\langle\mathrm{f}(\mathrm{z}) \mathrm{T}_{1} \mathrm{G}_{1}\right\rangle=\frac{1}{6}$

Model 2. Piecewise linear heating from below temperature profile:

$\left\langle\mathrm{f}(\mathrm{z}) \mathrm{W}_{1} \mathrm{~T}_{1}\right\rangle=\frac{1}{40}\left(8 \varepsilon^{4}-25 \varepsilon^{3}+20 \varepsilon^{2}\right)$

$\left\langle\mathrm{f}(\mathrm{z}) \mathrm{T}_{1} \mathrm{G}_{1}\right\rangle=\frac{1}{6}\left(-2 \varepsilon^{2}+3 \varepsilon\right)$

Model 3. Piecewise linear cooling from above temperature profile:

$$
\begin{aligned}
& \left\langle f(z) W_{1} T_{1}\right\rangle=\frac{1}{40}\left(8 \varepsilon^{4}-15 \varepsilon^{3}+10 \varepsilon\right), \\
& \left\langle f(z) T_{1} G_{1}\right\rangle=\frac{1}{6}\left(-2 \varepsilon^{2}+3 \varepsilon\right) .
\end{aligned}
$$

Model 4. Step function temperature profile:

$$
\begin{aligned}
& \left\langle\mathrm{f}(\mathrm{z}) \mathrm{W}_{1} \mathrm{~T}_{1}\right\rangle=\varepsilon^{4}-\frac{5}{2} \varepsilon^{3}+\frac{3}{2} \varepsilon^{2}, \\
& \left\langle\mathrm{f}(\mathrm{z}) \mathrm{T}_{1} \mathrm{G}_{1}\right\rangle=-\varepsilon^{2}+\varepsilon .
\end{aligned}
$$


Model 5. Inverted temperature profile:

$$
\left\langle\mathrm{f}(\mathrm{z}) \mathrm{W}_{1} \mathrm{~T}_{1}\right\rangle=\frac{1}{15}, \quad\left\langle\mathrm{f}(\mathrm{z}) \mathrm{T}_{1} \mathrm{G}_{1}\right\rangle=\frac{1}{6} .
$$

Model 6. Parabolic temperature profile:

$$
\left\langle\mathrm{f}(\mathrm{z}) \mathrm{W}_{1} \mathrm{~T}_{1}\right\rangle=\frac{1}{12}, \quad\left\langle\mathrm{f}(\mathrm{z}) \mathrm{T}_{1} \mathrm{G}_{1}\right\rangle=\frac{1}{6} .
$$

\section{Critical Rayleigh number for lower boundary rigid-isothermal and upper boundary free- adiabatic, no spin:}

The boundary conditions are

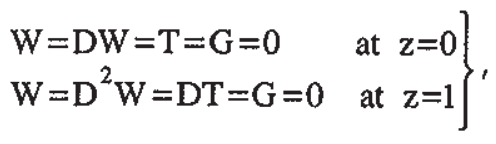

The trial functions satisfying (46) are

$$
\mathrm{W}_{1}=2 \mathrm{z}^{4}-5 \mathrm{z}^{3}+3 \mathrm{z}^{2}, \quad \mathrm{~T}_{\mathrm{l}}=\mathrm{z}(\mathrm{z}-2), \quad \mathrm{G}_{1}=\mathrm{z}(1-\mathrm{z})
$$

Substituting (47) in equation (27) and performing the integration, we get

$$
R=\frac{\left(5+2 a^{2}\right)}{36}\left\{\frac{28 y_{1}\left[\left(1+N_{1}\right) y_{2}+216 Q\right]-N_{1}^{2} y_{3}^{2}}{N_{1} N_{5} y_{3}\left\langle f(z) T_{1} G_{1}\right\rangle-28 y_{1}\left\langle f(z) W_{1} T_{1}\right\rangle}\right\},
$$

where

$$
\begin{aligned}
& y_{1}=N_{3}\left(10+a^{2}\right)+2 N_{1}, \\
& y_{2}=4536+432 a^{2}+19 a^{4}, \\
& y_{3}=126+13 a^{2} .
\end{aligned}
$$


For a given $f(z), R$ attains its minimum value $R_{c}$ at $a=a_{c}$.

The integrals $\left\langle f(z) W_{1} T_{1}\right\rangle$ and $\left\langle f(z) T_{1} G_{1}\right\rangle$ will have the following forms depending on the nature of basic temperature profile.

Model 1. Linear temperature profile:

$\left\langle\mathrm{f}(\mathrm{z}) \mathrm{W}_{\mathrm{I}} \mathrm{T}_{1}\right\rangle=\frac{-2}{35}, \quad\left\langle\mathrm{f}(\mathrm{z}) \mathrm{T}_{1} \mathrm{G}_{1}\right\rangle=\frac{-7}{60}$.

Model 2. Piecewise linear heating from below temperature profile:

$$
\begin{aligned}
& \left\langle f(z) W_{1} T_{1}\right\rangle=\frac{1}{280}\left(40 \varepsilon^{6}-210 \varepsilon^{5}+364 \varepsilon^{4}-210 \varepsilon^{3}\right) \\
& \left\langle f(z) T_{1} G_{1}\right\rangle=\frac{1}{60}\left(-12 \varepsilon^{4}+45 \varepsilon^{3}-40 \varepsilon^{2}\right)
\end{aligned}
$$

Model 3. Piecewise linear cooling from above temperature profile:

$$
\begin{aligned}
& \left\langle f(z) W_{1} T_{1}\right\rangle=\frac{1}{280}\left(40 \varepsilon^{6}-70 \varepsilon^{5}-56 \varepsilon^{4}+140 \varepsilon^{3}-70 \varepsilon^{2}\right) \\
& \left\langle f(z) T_{1} G_{1}\right\rangle=\frac{1}{60}\left(-12 \varepsilon^{4}+15 \varepsilon^{3}-20 \varepsilon^{2}-30 \varepsilon\right) .
\end{aligned}
$$

Model 4. Step function temperature profile:

$$
\begin{aligned}
& \left\langle\mathrm{f}(\mathrm{z}) \mathrm{W}_{1} \mathrm{~T}_{1}\right\rangle=\varepsilon^{6}-\frac{9}{2} \varepsilon^{5}+\frac{13}{2} \varepsilon^{4}-3 \varepsilon^{3}, \\
& \left\langle\mathrm{f}(\mathrm{z}) \mathrm{T}_{1} \mathrm{G}_{1}\right\rangle=-\varepsilon^{4}+3 \varepsilon^{3}-2 \varepsilon^{2} .
\end{aligned}
$$

Model 5. Inverted temperature profile:

$$
\left\langle f(z) W_{1} T_{1}\right\rangle=\frac{-19}{420}, \quad\left\langle f(z) T_{1} G_{1}\right\rangle=\frac{-1}{10} .
$$


Model 6. Parabolic temperature profile:

$$
\left\langle\mathrm{f}(\mathrm{z}) \mathrm{W}_{1} \mathrm{~T}_{1}\right\rangle=\frac{-29}{420}, \quad\left\langle\mathrm{f}(\mathrm{z}) \mathrm{T}_{1} \mathrm{G}_{1}\right\rangle=\frac{-2}{15} .
$$

\section{Results and Discussion}

In this paper we considered the following effects on the classical Rayleigh-Benard problem:

a) magnetic field inhibition of convection

b) micropolar fluid parameters, and

c) non-linear reference steady-state temperature distribution.

These three effects are, respectively, represented by the Chandrasekhar number $Q$, Micropolar fluid parameters $\left(N_{1}, N_{3}, N_{5}\right)$ and $\mathrm{f}(z)$ in the eigenvalue equation (27). The typical values of $Q$ are those traditionally chosen in Newtonian fluid problems (see Chandrasekhar [25], Platten and Legros [26]) and thermodynamic restrictions dictate the following permitted range values for $N_{1}, N_{3}$ and $M_{5}$ :

$0 \leq \mathrm{N}_{1} \leq 1, \quad 0 \leq \mathrm{N}_{3} \leq \mathrm{m}, \quad 0 \leq \mathrm{N}_{5} \leq \mathrm{n}$,

where $m$ and $n$ are finite, real numbers (see Siddheshwar and Pranesh [21]). One linear and five non-linear reference steady-state temperature profiles, i.e., $f(z)$ 's are chosen for study (see table (T)). It is observed that for the non-symmetric boundary combination critical Rayleigh number, $R_{c^{\prime}}$, holds the following inequality of the six models,

$$
\mathrm{R}_{\mathrm{c}_{4}}<\mathrm{R}_{\mathrm{c}_{3}}<\mathrm{R}_{\mathrm{c}_{2}}<\mathrm{R}_{\mathrm{c}_{6}}<\mathrm{R}_{\mathrm{c}_{1}}<\mathrm{R}_{\mathrm{c}_{5}} \mathrm{I}
$$

i.e., the step function is the most destabilizing basic temperature distribution and inverted parabolic is the most stabilizing basic temperature distribution. In the case of symmetric boundary combination the step-function and inverted parabolic temperature profile are respectively most destabilizing and stabilizing basic temperature distribution. For the non-symmetric boundaries in the case of isothermal temperature boundary condition the step function and inverted parabolic temperature profile are respectively most destabilizing and stabilizing basic temperature distribution. In the case of adiabatic temperature boundary conditions the stepfunction and heating from below temperature profiles are respectively most 
destabilizing and stabilizing basic temperature distribution. In the case of piecewise linear and step function profiles, the critical Rayleigh number $R_{c}$ depends on the thermal depth, $\varepsilon$, in addition to depending on the parameters of the problem. Tables (2) - (4) provide information on the critical thermal depth $\varepsilon_{c}$ that yields the critical eigenvalues for different boundary combinations in respect of all relevant basic temperature gradients.

Table (5): Why and how of the stabilizing / destabilizing effects of the suspension parameters $\mathrm{N}_{1}, \mathrm{~N}_{3}, \mathrm{~N}_{5}$ ?

\begin{tabular}{|c|c|l|}
\hline $\begin{array}{c}\text { Parameter } \\
0 \leq N_{3} \leq 1\end{array}$ & $\begin{array}{c}\text { Nature of effect } \\
\text { (as } N_{1} \text { increases) }\end{array}$ & $\begin{array}{l}\text { Physical reason } \\
\text { Increase in } N_{1} \text { indicates the increase } \\
\text { in the concentration of } \\
\text { microelements. These elements } \\
\text { consume the greater part of the } \\
\text { energy of the system in developing } \\
\text { the gyrational velocities of the fluid } \\
\text { and as a result the onset of } \\
\text { convection is delayed. }\end{array}$ \\
\hline $\begin{array}{c}N_{3} \\
\text { (m: finite,real) }\end{array}$ & $\begin{array}{c}\text { Destabilizing } \\
\text { (as } N_{3} \text { increases) }\end{array}$ & $\begin{array}{l}\text { Increase in } N_{3} \text {, decreases the couple } \\
\text { stress of the fluid which causes a } \\
\text { decrease in microrotation and hence } \\
\text { makes the system more unstable. }\end{array}$ \\
\hline $\begin{array}{c}N_{5} \\
\text { ( } \mathrm{n}: \text { finite, real) }\end{array}$ & $\begin{array}{c}\text { Stabilizing } \\
\text { (as } \mathrm{N}_{5} \text { increases) }\end{array}$ & $\begin{array}{l}\text { When } \mathrm{N}_{5} \text { increases, the heat } \\
\text { induced into the fluid due to these } \\
\text { microelements also increases, thus } \\
\text { reducing the heat transfer from } \\
\text { bottom to top. }\end{array}$ \\
\hline
\end{tabular}

The critical Royleigh number $R_{c}$ obtained using Rayleigh-Ritz technique for different values of $N_{1}, N_{3}, N_{5}$ and $Q$ are shown in figures (2) - (10). Figures (2) $-(4)$ are the plot of critical Rayleigh number $R_{c}$ versus coupling parameter $N_{1}$, couple stress parameter $\mathrm{N}_{3}$ and micropolar heat condition parameter $\mathrm{N}_{5}$ for different values of Chandrasekhar number $Q$ and for different non-uniform temperature gradients for different velocity boundary combination and isothermal temperature boundaries. Figures (5) - (7) are the plot of critical Rayleigh number $R_{c}$ versus $N_{1}, N_{3}$ and $N_{5}$ for different values of Chandrasekhar number $Q$ and for different non-uniform 
temperature gradients for different velocity boundary combination and adiabatic temperature boundaries. Figure ( 8 ) is the plot of critical Rayleigh number $R$ versus $N_{1}, N_{3}$ and $N_{5}$ for different values of Chandrasekhar number $Q$ and for different non-uniform temperature gradients for lower rigid isothermal and upper free adiabatic boundary combination. Figures $(9)-(10)$ are the plot of critical Rayleigh number $R_{c}$ versus $N_{1}, N_{3}$ and $N_{5}$ with respect to linear temperature profile for different velocity boundary combination and for isothermal and adiabatic temperature condition respectively. From these figures, we obseve that as $N_{1}$ increases $R_{c}$ also increases, $N_{3}$ increases $R_{c}$ decreases and $N_{5}$ increases $R_{c}$ also increases, the reason for the increase and decrease of $R_{c}$ is given in table (5). From the figures (9) and (10) we observe that the results of isothermal and adiabatic boundaries are qualitatively similar. Also we observe from these figures $R_{c}^{R R}>R_{c}^{R F}>R_{c}^{F F}$ where the superscripts corresponds to the three velocity boundary combinations.

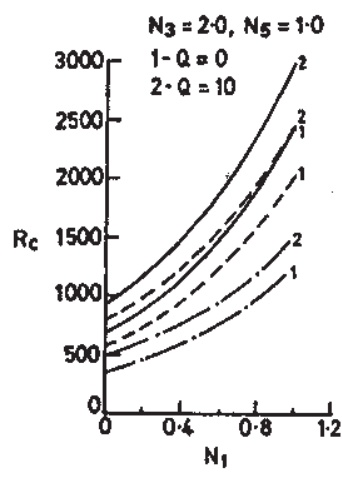

(a)

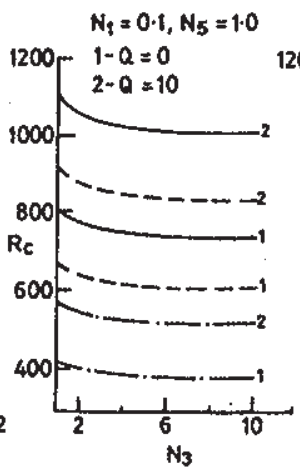

(b)

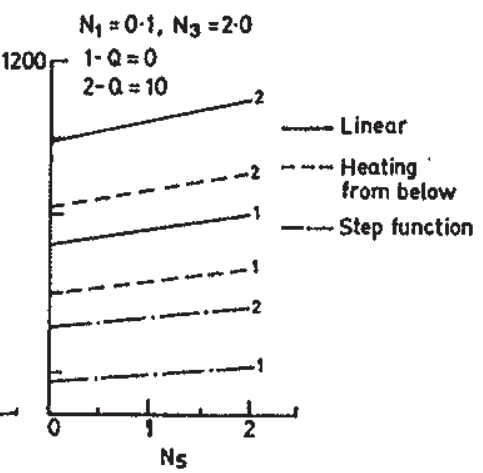

(c)

Fig. 2. Plot of critical Rayleigh number $R_{1} V_{s}$. (c) Coupling parameter $N_{1}$ (b) Couple stress parameter $\mathrm{N}_{3}$ and (c) Micropolar heat conduction parameter $\mathrm{N}_{5}$ with respect to free-free isothermal boundary for different values of Chandrasekhar number $Q$ and different Non-uniform basic temperature gradients. 


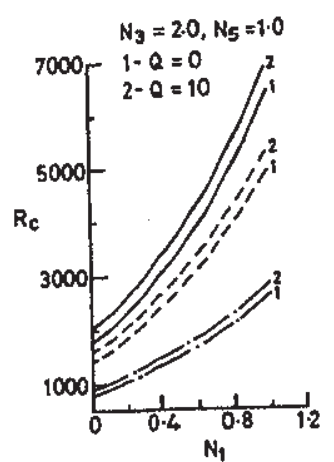

(a)

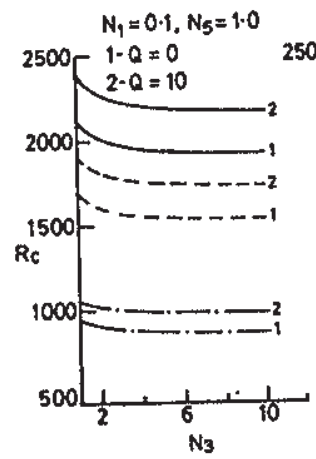

(b)

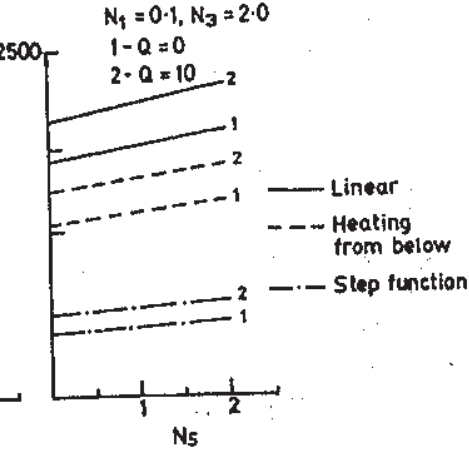

(c)

Fig. 3. Plot of $R_{c} V_{s}$. (a) $N_{1}$, (b) $N_{3}$ and (c) $N_{5}$ with respect to Rigid-Rigid Isothermal boundary, for different values of $Q$ and different non-uniform basic temperature gradients.

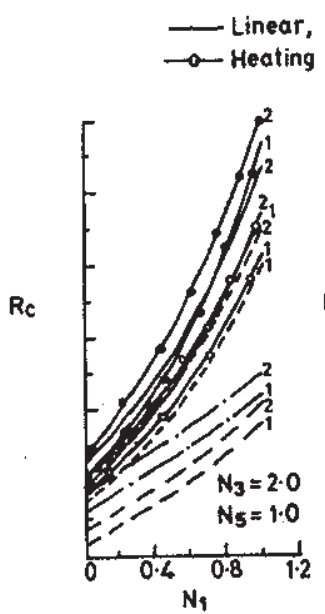

(a)
- Cooling from above, - - Step function

$\rightarrow-$ Inverted parabolic, ---- Parabolic

$1-Q=0$

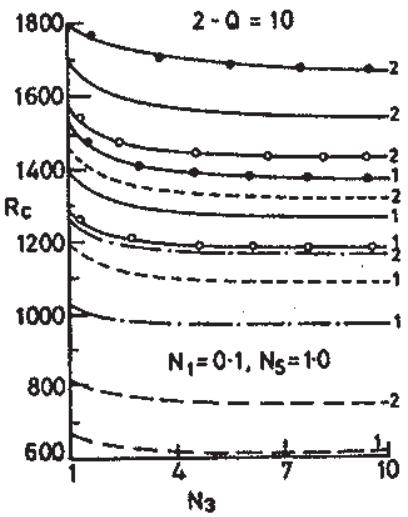

(b)

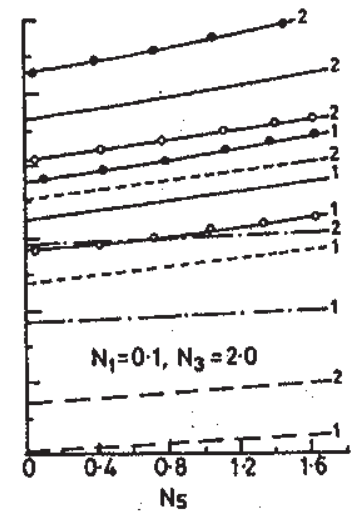

(c)

Fig. 4. Plot of $R_{c} V_{s}$. (a) $N_{1}$, (b) $N_{3}$ and (c) $N_{5}$ with respect to Rigid-Free isothermal boundary, for different values of $Q$ and different non-uniform basic temperature gradients. 


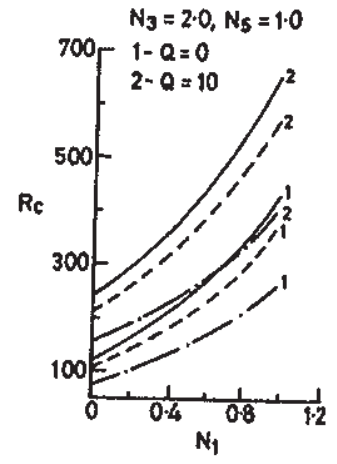

(a)

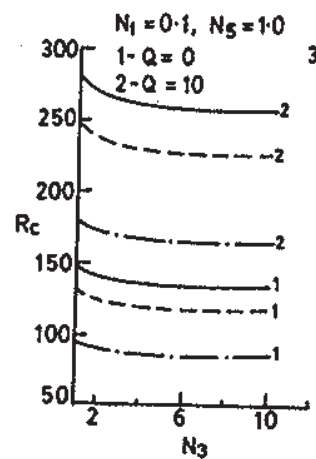

(b)

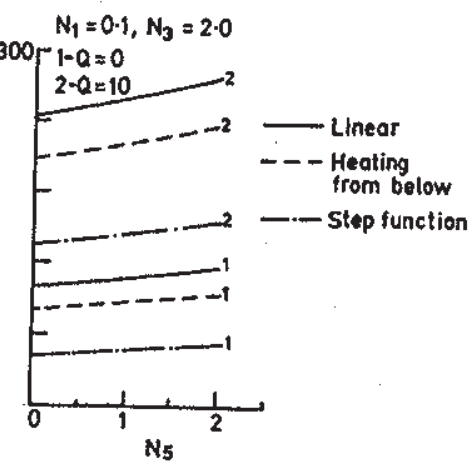

(c)

Fig. 5. Plot of $R_{c} V_{s}$. (a) $N_{1}$, (b) $N_{3}$ and (c) $N_{5}$ with respect to Free-Free Adiabatic boundary, for different values of $Q$ and different non-uniform basic temperature gradients.

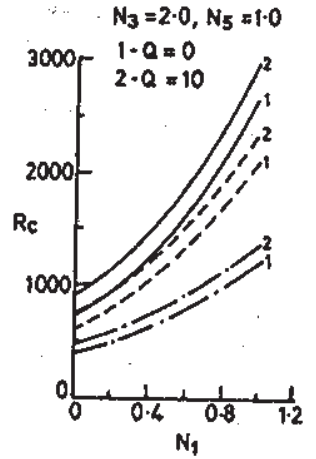

(a)

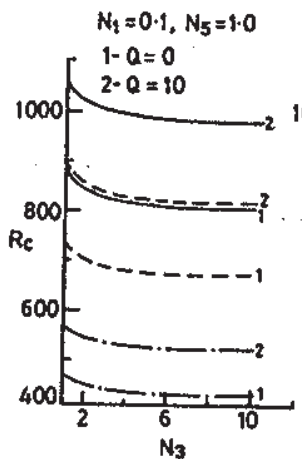

(b)

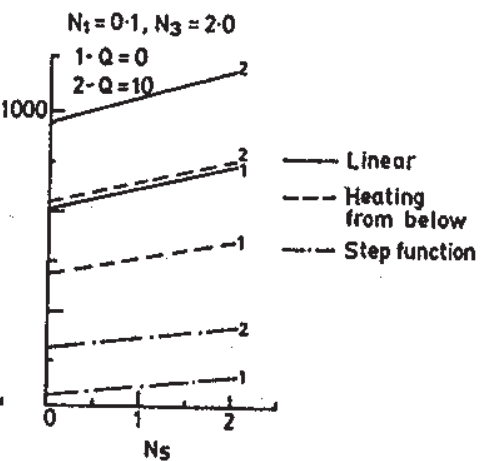

(c)

Fig. 6. Plot of $R_{c} V_{s}$. (a) $N_{y}$ (b) $N_{3}$ and (c) $N_{5}$ with respect to Rigid-Rigid Adiabatic boundary, for different values of $Q$ and different non-uniform basic temperature gradients. 


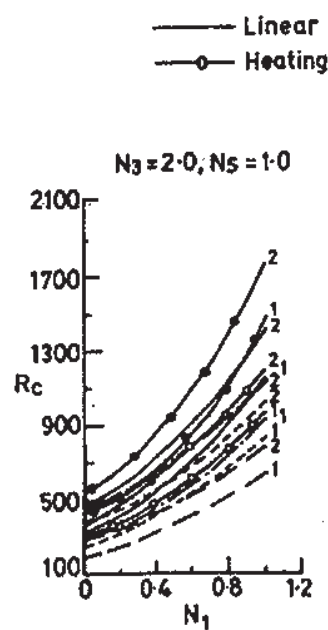

(a)

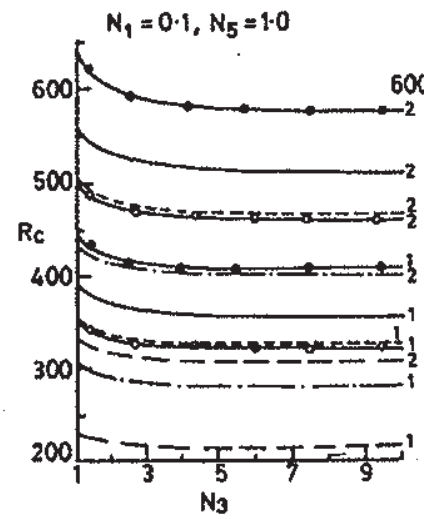

(b)
- - Step function

Parabolic

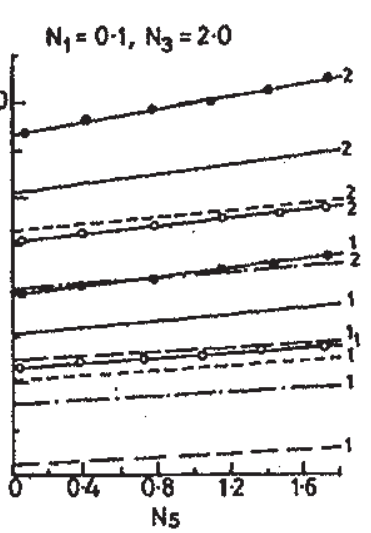

(c)

Fig. 7. Plot of $R_{c} V_{s}$. (a) $N_{1}$ (b) $N_{3}$ and (c) $N_{5}$ with respect to Rigid-Free Adiabatic boundary, for different values of $Q$ and different non-uniform basic temperature gradients.

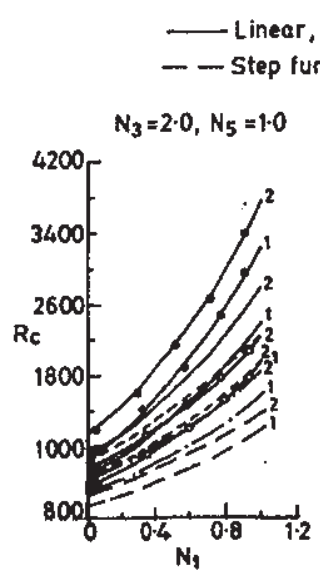

(a)

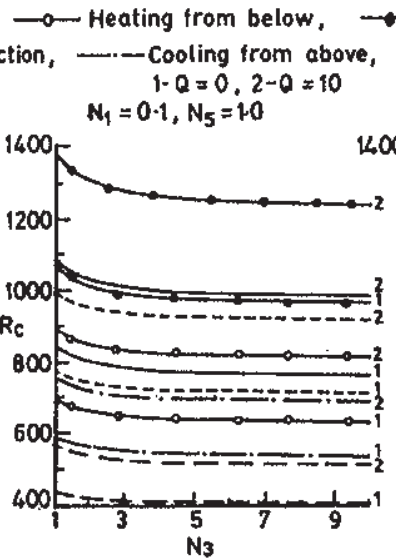

(b)

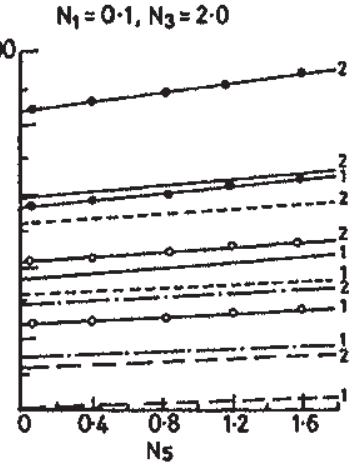

(c)

Fig. 8. Plot of $R_{c} V_{s}$. (a) $N_{1}$ (b) $N_{3}$ and (c) $N_{5}$ with respect to Lower Rigid Isothermal and Upper Free Adiabatic for different values of $Q$ and different non-uniform basic temperature gradients. 


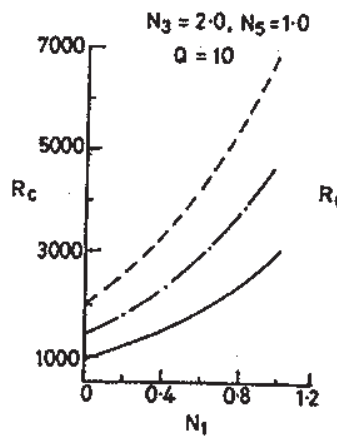

(a)

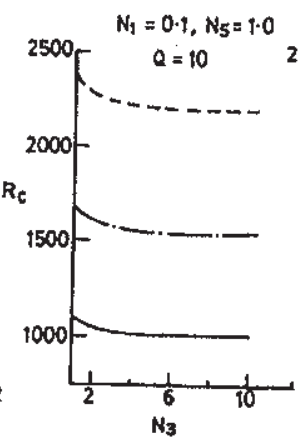

(b)

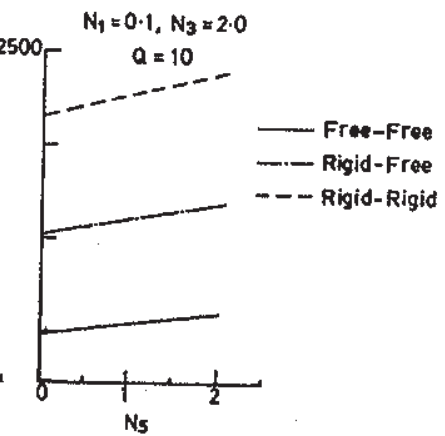

(c)

Fig. 9. Plot of $R_{c} V_{s}$. (a) $N_{1}$, (b) $N_{3}$ and (c) $N_{5}$ for different velocity boundary Combinations (Isothermal) with respect to linear temperature profiles.

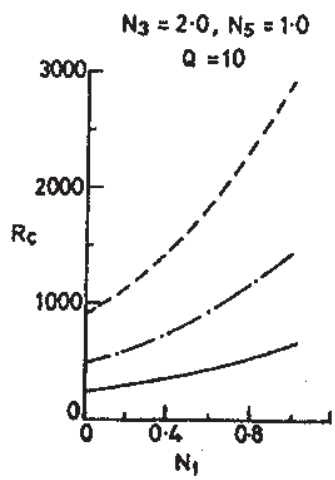

(a)

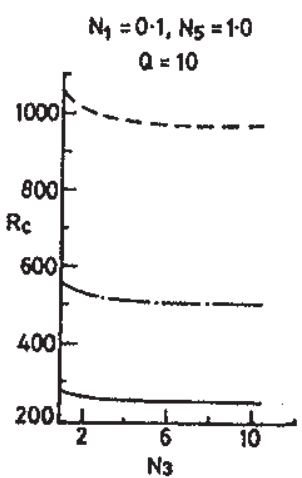

(b)

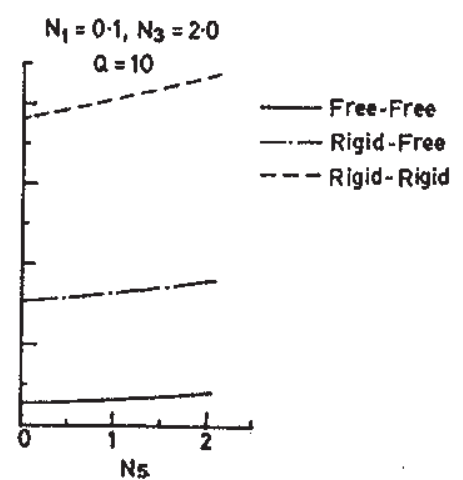

(c)

Fig. 10. Plot of $R_{c} V_{s}$. (a) $N_{1}$ (b) $N_{3}$ and (c) $N_{5}$ for different velocity boundary Combinations (Adiabatic) with respect to linear temperature profiles. 
The effects of $N_{1}, N_{3}$ and $N_{5}$ on $R_{c}$ is true both in the presence and absence of a magnetic field. When the strength of the magnetic field increases, the system becomes stable, a result which is as seen in the case of classical fluid. On the other hand, when the microrotation and the magnetic field are simultaneously present, the stabilizing effect of $N_{1}$ is reduced, being counteracted by the magnetic field. Chandrasekhar [25] has noticed the similar phenomenon, when the system is subject to both rotation and magnetic field. The above phenomenon can be physically explained as follows:

When the magnetic field strength permeating the medium is considerably strong, it induces viscosity into the fluid, and the magnetic lines as distorted by convection. Then these magnetic lines hinder the growth of disturbances, leading to the delay in the onset of instability. However, the viscosity produced by the magnetic field lessens the rotation of the fluid particles, thus controlling the stabilizing effect of $N_{1}$.

From the calculations it has been found that the critical wave number is, in general, insensitive to the changes in the micropolar parameters but is influenced by the magnetic field. A strong magnetic field succeeds in inducing only the coupling number $N_{1}$ into influencing $a_{c}^{2}$.

\section{Conclusion}

The above results indicate that the externally applied magnetic field is an effective means of controlling Rayliegh-Benard convection in electrically conducting micropolarfluids. The results suggest that Rayleigh-Benard convection in Newtonian fluids may be delayed by adding micron sized electrically inert suspended particles. Further, by creating conditions for an appropriate basic temperature gradient we can also make a priori decision on advancing or delaying convection.

\section{Acknowledgement}

The author would like to thank Dr. Pradeep G. Siddheshwar for suggesting the problem and for many stimulating discussions, and to the management of Christ College for their support in completing this work. 


\section{References}

1. Currie, I. G.: The effect of heating rate on the stability of stationary fluids, Journal of Fluid Mechanics, 29, 337-347, (1967).

2. Nield D. A.: The onset of transient convective instability, J Fluid Mech., 71, 3-11, (1975).

3. Lebon $G$, Cloot A.: Effects of non-uniform temperature gradients on Benard-Marangoni instability. J. Non-equin. Thermodyn., 6, 15-30, (1981).

4. Rudraiah N, Chandna O. P., Garg M. R.: Effect of non-uniform temperature gradient on magneto-convection driven by surface tension and buoyancy. Ind. J. Technology., 24, 279 . 284 , (1986).

5. Eringen A. C.: Simple microfluids with stretch. Int. J. Engg. Sci, 7, 115-127, (1969).

6. Eringen A. C.: Micropolar fluids with stretch. Int. J. Engg. Sci., 7, 1 15-127, (1986).

7. Eringen A. C.: Micropolar theory of liquid crystals. In: Johnson, J. F., Porter R. S. (Eds), Liquid crystals and ordered fluids, 3, Plenum Publishing Co., 1978. 8. Power H.: Bio-Fluid Mechanics, Advances in fluid mechanics, W.I.T. Press, UK, 3, 336,
(1995).

9. Lukaszewicz: Micropolar fluid theory and applications, Birkhauser Boston, M. A., USA, 1998.

10. Eringen A. C.: Microcontinuum fluid theories, Springer Verlag, 1999.

11. Chandra K.: Instability of fluids heated from below. Proc. Roy. Soc. London A., 164, 231. 242 , (1938).

12. Datta A, B., Sastry V. U. D.: Thermal instability of a horizontal layer of micropolar fluid heated from below. Int. J. Engg. Sci., 14, 631-637, (1976).

13. Bhattacharya S. P., Jena S. K.: Thermal instability of a horizontal layer of micropolar fluid with heat source. Int. J. Engg. Sci., 23, 13-26, (1984).

14. Payne L. E., Straughan B.: Critical Rayleigh number for oscillatory and non-linear convection in an isotropic thermomicropolar fluid. Int. J. Engg. Sci., 27, 827-836, (1989).

15. Siddheshwar P. G., Pranesh S.: Magnetoconvection in a micropolar fluid. Int. J. Engg. Sci., 36, 1173-1181, (1998).

16. Siddheshwar P. G., Pranesh S.: Effect of a non-uniform basic temperature gradient on Rayleigh-Benard convection in a micropolar fluid. Int. J. Engg. Sci., 36, 1183-1196.
(1998).

17. Siddheshwar P. G., Pranesh S.: Effect of temperature / gravity modulation on the onset of magneto-convection in a weak electrically conducting fluids with intenal angular momentum. JMMM, 192, 159-176, (1999).

18. Siddheshwar P. G., Pranesh S.: Effect of temperature / gravity modulation on the onset of magneto-convection in an electrically conducting fluids with intenal angular momentum. JMMM, 219, L153 - L162. (2000). 
19. Siddheshwar P. G., Pranesh S.: Effects of non-uniform temperature gradients and magnetic field on the onset of convection in fluids with suspended particles under microgravity conditions, Indian Joumal of Engineering and Mateials Sciences, 8, 77-83 (2001).

20. Siddheshwar P. G., Pranesh S.: Suction-Injection effects on the onset of Rayleigh-BenardMarangoni convection in a fluid with suspended particles, Acta Mechanica, 152, 241-252 (2001).

21. Siddheshwar P. G., Pranesh S.: Magnetoconvection in fluids with suspended particles under $\mathrm{lg}$ and $\mathrm{mg}$, International Journal of Aerospace Science and Technology, 6, 105-114 (2002).

22. Pranesh S.: Effects of Suction-Injection-Combination $(\mathrm{SIC})$ on the onset of Rayleigh-Benard magnetoconvection in a fluid with suspended particles, International Journal of Engineering Sciences, 41, 1741-1766 (2003).

23. Siddheshwor P. G., Pranesh S.: Linear and weakly non-linear analyses of convection in a micropolar fluid, Hydrodynamics VI-Theory and applications Cheng \& Yeow (eds) 487 493 (2005).

24. Rudraish N., Siddheshwar P. G.: Effect of non-uniform basic temperature gradient on the onset of Marangoni convection in a fluid with suspended particles. J. Aerospace Sci. and Tech. 4(8), 517-523, (1999).

25. Chandrasekhar S.: Hydrodynamic, Hydromagnetic Stability, Dover, New York, 1961.

26. Platten J. K., Legros J. C.: Convection in liquids, Springer Berlin, 1984. 